\title{
Research on Microgrid Optimization Based on Simulated Annealing Particle Swarm Optimization
}

\author{
Shuyi $\mathrm{Li}^{1, *}$, Xifeng Zhou ${ }^{1}$, and Qiangang Guo ${ }^{1}$ \\ ${ }^{1}$ Automation,\&Artificial Intelligence Institute, Nanjing University of Posts and Telecommunications, Nanjing, China
}

\begin{abstract}
Based on the pursuit of different goals in the operation of the microgrid, it is not possible to meet the lowest cost and the least pollution at the same time. From the perspective of economy and environmental protection, a microgrid model including photovoltaic power generation, wind power generation, micro gas turbine, fuel cell and energy storage device is proposed. This paper establishes a comprehensive benefit objective function that considers both microgrid fuel cost, maintenance management cost, depreciation cost, interaction cost with public grid and pollutant treatment cost. In order to avoid the defect that the traditional particle swarm optimization algorithm is easy to fall into the local optimal solution, this paper uses the combination of simulated annealing algorithm and particle swarm optimization algorithm to compare with the traditional particle swarm optimization algorithm to obtain a more suitable method for microgrid operation. Finally, a typical microgrid in China is taken as an example to verify the feasibility of the algorithm.
\end{abstract}

\section{Preface}

The form of distributed power in the microgrid is diverse. There are photovoltaic and wind power generation devices that make full use of natural energy. There are also micro gas turbines and fuel cells that use clean energy. If necessary, there are also traditional fossil fuel power generation devices. Microgrid also has access to energy storage devices and Public grids to increase the reliability of power usage. The combination of multiple distributed power sources and the output method can solve different power demand, Under the premise of ensuring reliable power supply reliability, scientific distribution can maximize economic benefits and minimize environmental pollution. The focus of the current discussion.

In this paper, some high-altitude or island demonstration areas in China are carrying out research on the practical needs of grid-connected distributed power microgrids, using intelligent algorithms combined with simulated annealing algorithm and particle swarm optimization algorithm to optimize the environmental economic optimization of microgrid systems. Explore. For one thing, from the perspective of micro-grid economy, the fuel consumption cost, maintenance management cost, depreciation loss cost and power interaction cost of public grid are taken into account; for another thing, from the perspective of micro-grid environmental protection, The unit treatment cost of pollutant gas emissions (including $\mathrm{CO}, \mathrm{CO}_{2}, \mathrm{SO}_{2}$, and $N O_{x}$ ), establish a comprehensive benefit optimization model that considers both aspects. Under the premise of ensuring the safety and stability of the microgrid and meeting the needs of users, the example is used to solve and demonstrate.

\section{Grid-connected microgrid optimization mathematical model}

\subsection{Grid-connected microgrid structure model}

The first step in solving the environmental economic optimization problem of the microgrid is to establish a mathematical model. The common grid-connected microgrid structure is shown in Figure 1 [1-2]:

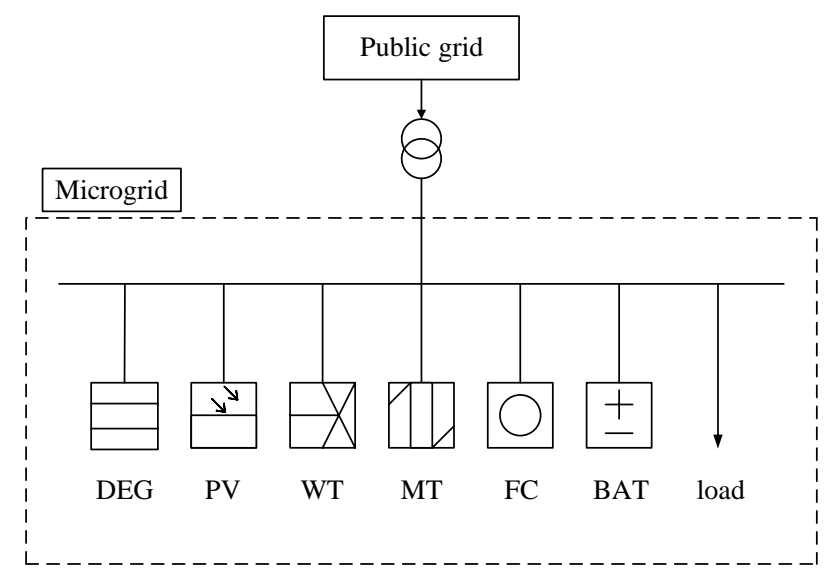

Fig. 1. Grid-connected microgrid structure model

\footnotetext{
* Corresponding author: 295680662@qq.com
} 
The grid-connected microgrid generally includes: a micro power supply device, a user load, an energy storage device, and the like. The micro power supply devices mentioned in this paper are: Photovoltaic power generation system (PV), Wind turbine (WT), Micro gas turbine (MT), Fuel cell (FC) And diesel generators (Diesel generators, DEG for short). The energy storage device in this model considers the more common leadacid batteries (BAT), and the load refers to the daily load demand in life. When solving the micro-grid optimization scheduling problem, a mathematical model is built according to the performance of each device to calculate the working condition of each unit.

\subsection{Grid-connected microgrid optimization description}

Although the situation discussed in this paper is the optimal operation of the grid-connected microgrid, it is best to ensure that the microgrid (such as high altitude or island demonstration area) is self-sufficient in power supply. When the microgrid cannot meet the daily load demand, the power of the large grid is considered. Because solar photovoltaic devices and wind power generation devices that rely on natural energy to generate electricity are relatively economical, recyclable, clean and pollution-free, are the most ideal power generation methods, and are the top priority of power generation equipment in this paper, that is, when photovoltaics and When the maximum power of wind power is used, the second-level micro gas turbine and fuel cell are used to generate electricity as much as possible. The fuel used by the two is a sustainable clean energy, but it is also constrained by the power constraints and climbing rate of each power generation unit. Such restrictions, so the third level of power generation equipment large energy grid and energy storage device also need to participate. Because the operation of the microgrid is a complex system that cooperates with each other, it is necessary to consider the instability and volatility of photovoltaic and wind treatment, and consider the practical problems of the treatment of the entire system's polluted gases. In the case of ensuring stable operation of the system, It can be combined with modern intelligent algorithms to calculate the best micro-grid operation mode with the most reasonable and comprehensive benefits.

\subsection{Microgrid optimization objective function}

In this paper, the optimal operation of microgrid is considered economic operation, followed by environmental protection, and finally consider the comprehensive benefits of the two [3].

(I) Objective function one: economic operation

$\min C_{1}=\sum_{t=1}^{T}\left(C_{f}(t)+C_{m a}(t)+C_{\text {dep }}(t)+C_{\text {gov }}(t)+k C_{\text {grid }}(t)\right)(1)$

Where: $C_{1}$ is only considering the operating cost of the microgrid economic operation; $\sum_{t=1}^{T} \mathrm{O}$ refers to the calculation of the average daily operating cost, $T=24$, ie
1-24 hours Operating cost; $C_{f}$ is the fuel cost per kWh converted; $C_{m a}$ is the maintenance management cost per $\mathrm{kWh}$ converted to each power unit; $C_{\text {dep }}$ is the depreciation loss of each power unit; $C_{g o v}$ is the government subsidy amount for clean energy; $k C_{\text {grid }}$ refers to the interaction cost with the large power grid. When purchasing electricity from the large power grid, $\mathrm{k}>0$; when selling electricity to the large power grid, $\mathrm{k}<0$. among them:

$$
C_{f}(t)=\sum_{i=1}^{N} C_{\text {fuel }} \times \frac{1}{L H V} \times \sum_{t=1}^{T} \frac{P_{i}(t)}{\eta_{i}(t)}
$$

Where: fuel cost $C_{f}(t)$ mainly refers to the cost of natural gas consumption (yuan); i from 1 to $\mathrm{N}$ represents the number of micro-power in the micro-grid; $C_{\text {fuel }}$ refers to the price of fuel (yuan $/ \mathrm{m}^{3}$ ), the price of natural gas in this paper 3.75 yuan $/ \mathrm{m}^{3} ; L H V$ refers to the low calorific value of natural gas $\left(\mathrm{kw} \cdot \mathrm{h} / \mathrm{m}^{3}\right)$, this paper takes $9.7 \mathrm{kw} \cdot \mathrm{h} / \mathrm{m}^{3} ; P_{i}(t)$ refers to the output power of the $\mathrm{i}$-th power generation unit at time $\mathrm{t}(\mathrm{kw})$; $\eta_{i}(t)$ refers to the output operating efficiency (\%) of the $\mathrm{i}$-th power generating unit at time $\mathrm{t}$.

$$
C_{i, m a}(t)=\sum_{i=1}^{N} M A_{i}\left(P_{i, t}\right)=\sum_{i=1}^{N} K_{M A_{i, t}} \times P_{i, t}
$$

Where: $C_{i, m a}(t)$ refers to the maintenance management cost (yuan) of the $\mathrm{i}$-th power generation unit at time $\mathrm{t}$; $P_{i, t}$ refers to the output power (kw) of the i-th power generation unit at time $\mathrm{t} ; K_{M A_{i, t}}$ refers to the maintenance management cost coefficient (yuan $/ \mathrm{kw} \cdot \mathrm{h}$ ) of the $\mathrm{i}$-th power generation unit at time $t$.

$$
C_{i, d e p}(t)=\sum_{i=1}^{N} D E P_{i}\left(P_{i, t}\right)=\sum_{i=1}^{N} \frac{A D C_{i}}{P_{N, i} \times 8760 \times c f_{i}} \times P_{i, t}
$$

Where: $C_{i, d e p}(t)$ refers to the depreciation loss cost (yuan) of the i-th power generation unit at time $\mathrm{t}$; $A D C_{i}$ refers to the annual average depreciation cost of the i-th power generation unit (yuan); $P_{N, i}$ refers to the maximum output power $(\mathrm{kw})$ of the i-th power generation unit; 8760 refers to 8760 hours of the year; $c f_{i}$ refers to the capacity factor $(\%)$ of the i-th power generation unit.

(II) Objective function two: environmentally friendly operation

$$
\min C_{2}=\sum_{t=1}^{T} \sum_{j=1}^{J} \alpha_{j}\left(\sum_{i=1}^{I} \beta_{i j} P_{i t}+\beta_{m j} P_{m t}\right)
$$

Where: $C_{2}$ is only considering the operating cost (yuan) of the microgrid environmental protection 
operation, mainly considering the treatment cost of the polluted gas; $\mathrm{j}$ is the $\mathrm{j}$-class pollutant gas (including the $\mathrm{CO}, \mathrm{CO}_{2}, \mathrm{SO}_{2}$ and $\mathrm{NO}_{x}$ derivatives generated in the microgrid and the large grid); $\alpha_{j}$ refers to the processing cost of the jth class of polluting gases (yuan $/ \mathrm{kw}$ ); $\beta_{i j}$ and $\beta_{m j}$ refer to the emissions of the jth class of polluting gases in the microgrid power generation unit and The emission coefficient of unit power is converted in the large power grid; $P_{i t}$ and $P_{m t}$ refer to the current actual working power $(\mathrm{kw})$ of the microgrid power generating unit and the large power grid at time $t$.

\section{(III) Objective function three: comprehensive operation}

$$
\left\{\begin{array}{c}
\min C_{3}=\left[C_{1}, C_{2}\right] \\
C_{3}=\lambda_{1} C_{1}+\lambda_{2} C_{2} \\
\lambda_{1}+\lambda_{2}=1
\end{array}\right.
$$

Where: $C_{3}$ is a comprehensive consideration of the economical and environmentally friendly operation cost of the micro-grid (yuan), and maintaining a certain balance between economy and environmental protection is the best optimization result pursued in this paper[4]. This paper uses the linear weight assignment method. Turning multi-objective issues of economics and environmental protection into a single-target problem. The weight coefficients $\lambda_{1}$ and $\lambda_{2}$ are not obtained by subjective conjecture, nor are they randomly generated by computer. In this paper, the value of the adaptive function is selected according to the variation law of the objective function. $\lambda_{1}=0.43$ was selected by experiment.

\subsection{Optimization of operating constraints}

We must ensure the safety, stability and feasibility of microgrid operation. Based on the power balance in the microgrid, all power generation units, energy storage equipment, transmission lines, etc. need to meet certain constraints.

1) Grid power balance constraints

$$
\sum_{i=1}^{N} P_{i}(t)+P_{\text {bat }}(t)+P_{\text {grid }}(t)=P_{l}(t)
$$

Where: $P_{i}(t)$ represents the power generation $(\mathrm{kw})$ of the $\mathrm{i}$-th power generating unit currently working at time $\mathrm{t} ; P_{\text {bat }}(t)$ represents the power $(\mathrm{kw})$ emitted by the energy storage device, and if it is a negative number, it is the energy storage of the microgrid Device charging; $P_{\text {grid }}(t)$ indicates the power $(\mathrm{kw})$ purchased from the public grid. If it is negative, the microgrid sells electricity to the public grid; $P_{l}(t)$ indicates the load power $(\mathrm{kw})$ required by the user at the current time.

2) Output constraints of each power generation unit in the microgrid

$$
P_{\text {imin }}(t) \leq P_{i}(t) \leq P_{\text {imax }}(t)
$$

3) Controlled power generation unit climbing rate constraint in microgrid

$$
\left\{\begin{array}{c}
\left|P_{i, \text { up }}(t)-P_{i, \text { up }}(t-1)\right| \leq R_{i, \text { up }} \cdot \Delta t \\
\left|P_{i, \text { down }}(t)-P_{i, \text { down }}(t-1)\right| \leq R_{i, \text { down }} \cdot \Delta t
\end{array}\right.
$$

Where: $P_{i, u p}(t)$ represents the active power $(\mathrm{kw})$ of the i-th power generating unit currently working at time t; $R_{i, u p}$ represents the increased active power of the i-th power generating unit currently in operation Power limit (kw).

4) Microgrid tie line transmission power constraint

$$
P_{\text {line }, \min } \leq P_{\text {line }} \leq P_{\text {line }, \max }
$$

Where: $P_{\text {line }}$ represents the line transmission capacity $(\mathrm{kw})$ between the microgrid and the distribution network.

5) Energy storage device charging and discharging capacity constraint

$$
\begin{gathered}
S_{\text {SOCmin }} \leq S_{\text {SOC }}(t) \leq S_{\text {SOCmax }} \\
\left\{\begin{array}{c}
P_{\text {BESSin }}(t) \leq P_{\text {BESSin,max }} \\
P_{\text {BESSout }}(t) \leq P_{\text {BESSout,max }}
\end{array}\right.
\end{gathered}
$$

Where: $S_{S O C}(t)$ represents the state of charge of the energy storage device at time $\mathrm{t} ; P_{B E S S i n}(t)$ and $P_{\text {BESSout }}(t)$ respectively represent the charge and discharge power of the energy storage device at time $t$, and a certain upper limit is required[5].

6) Exchange power constraints with public grids

The annual exchange capacity of the microgrid and the external grid generally does not exceed $50 \%$ of the annual electricity consumption. This paper takes into account the black start capability of the microgrid, so the annual exchange capacity of the constrained microgrid and the public grid does not exceed $40 \%$ of the annual electricity consumption.

$$
\sum_{t=1}^{T} P_{\text {grid }}(t) \leq 0.4 \sum_{t=1}^{T} \sum_{i=1}^{N} P_{i}(t)
$$

Where: $\sum_{t=1}^{T} P_{\text {grid }}(t)$ indicates the exchange power of the microgrid and the large power grid 24 hours a day, mainly refers to the purchased power $(\mathrm{kw})$.

\section{Improved simulated annealing particle swarm optimization algorithm}

\subsection{Traditional particle swarm optimization}

The idea of Particle Swarm Optimization (PSO) is derived from the predation behavior of birds/fish groups, 
simulating the foraging behavior of birds flying in the air. PSO uses the way of cooperation between bird and birds to find out the optimal purpose that where is the food and it is based on an optimization algorithm of Swarm Intelligence. The algorithm flow chart is as follows:

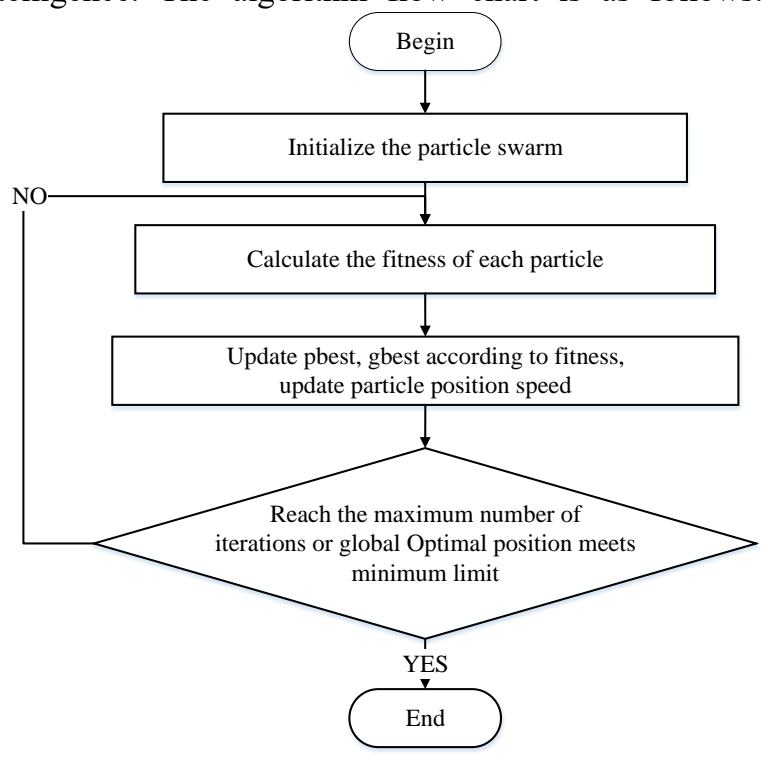

Fig. 2. Traditional particle swarm algorithm flow chart

\subsection{Particle swarm optimization algorithm based on simulated annealing algorithm.}

The Simulate Anneal (SA) algorithm is also a Greedy algorithm, but it introduces random factors in the search process compared to the ordinary Greedy algorithm. That is to say, it has a certain probability to accept a solution that is worse than the current solution, so you can jump out of this extreme value, that is, get rid of the local optimal solution, and find the global optimal solution. This algorithm can solve the defect that the particle swarm algorithm is easy to fall into the local optimal solution[6]. The algorithm flow chart is as Fig. 3.

\subsubsection{Particle Swarm Optimization Algorithm Based on Simulated Annealing Algorithm}

The particle swarm optimization algorithm (SAPSO) based on simulated annealing algorithm proposed in this paper introduces the idea of probability jump in simulated annealing algorithm, so that SAPSO algorithm not only accepts better solution, but also has a certain probability to try to accept the difference. Solution, consider more possibilities, make the particle population's motion behavior more reasonable, and get a better microgrid operation mode. The SAPSO algorithm operation process is as follows:

a) Initialization parameters: inertia weight $\omega$, learning factor $c_{1}, c_{2}$, and annealing speed $\delta$.

b) Randomly generate a population containing $\mathrm{m}$ particles, randomly initializing the velocity and position of the particle.

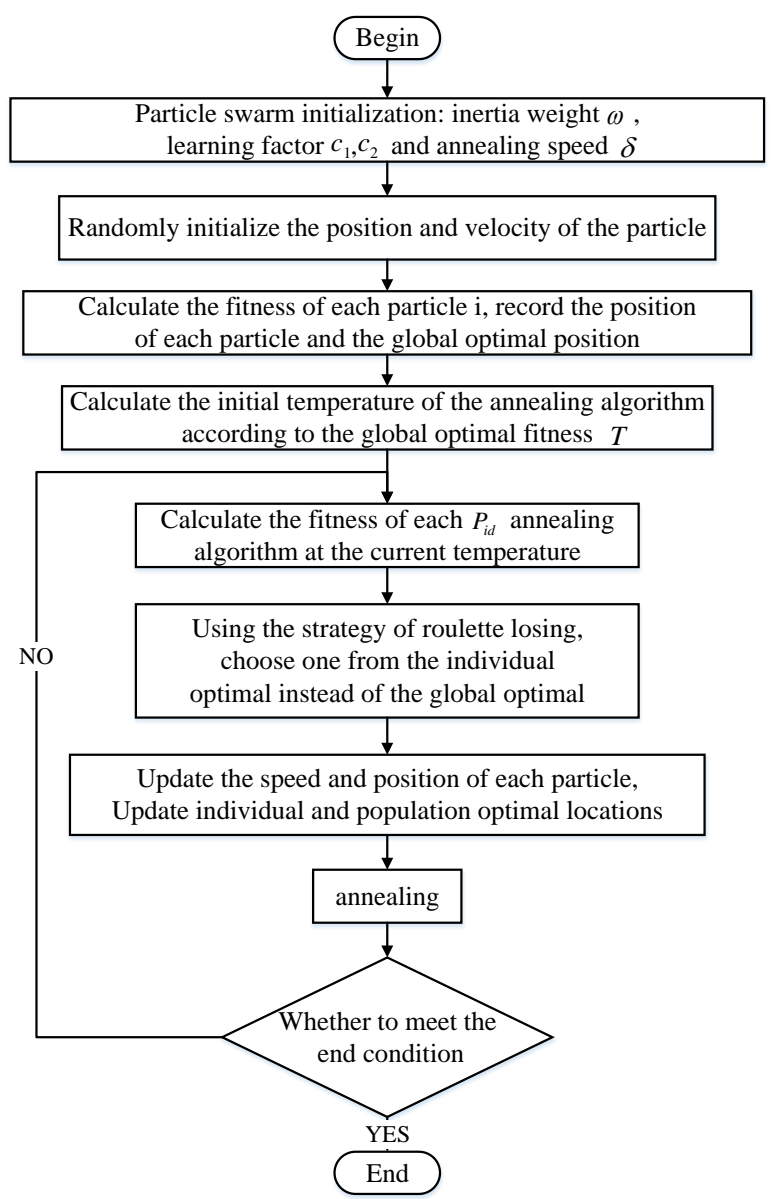

Fig. 3. Flow chart of particle swarm algorithm for simulated annealing algorithm

c) Calculate the fitness $(x(i))$, fitness $(x(i))$ of each particle $\mathrm{i}$ as the fitness function, that is, the objective function, abbreviated as $f(x)$, and record the position $P_{i d}$ of each (i-th) particle. , global optimal position $P_{p d}$, fitness $f\left(P_{i d}\right)$ and global optimal fitness $f\left(P_{p d}\right)$.

d) The initial temperature $\mathrm{T}$ of the annealing algorithm is calculated according to the global optimal fitness $f\left(P_{p d}\right)$

e) The $f_{S A}(\mathrm{x})$ function is a function to calculate the fitness of the annealing algorithm. The annealing algorithm fitness of each $P_{i d}$ at the current temperature T:

$$
f_{S A}\left(P_{i d}\right)=\frac{e^{-\frac{f\left(P_{i d}\right)-f\left(P_{p d}\right)}{T}}}{\sum_{i=1}^{n} e^{-\frac{f\left(P_{i d}\right)-f\left(P_{p d}\right)}{T}}}
$$

f) Apply roulette[7] to select the idea of genetic algorithm, choose one from the individual optimal position $P_{i d}$ instead of the global most position $P_{p d}$, denoted as $P_{r d}$, the algorithm is as follows:
A. $\quad p$ Bet $=\operatorname{rand} O(p$ Bet is a random number from 0 to 1 ). 
B. Calculate the cumulative probability $\operatorname{comfit}(j)=\sum_{i=1}^{j} f_{S A}\left(P_{i d}\right)$ according to the annealing algorithm fitness $f_{S A}\left(P_{i d}\right)$.

C. According to the cumulative probability, the individual optimal position $P_{r d}$ of the r-th particle satisfying the condition is selected instead of the global optimal position $P_{p d}$. The condition is: comfit $(r-1)<p$ Bet $<$ comfit $(r)$.

g) Replace $P_{p d}$ with $P_{r d}$, bring in the particle swarm formula, and update the speed of each particle as follows:

$v_{i d}^{k+1}=\omega v_{i d}^{k}+c_{1} r_{1}\left(p_{i d}^{k}-x_{i d}^{k}\right)+c_{2} r_{2}\left(p_{r d}^{k}-x_{i d}^{k}\right)(15)$

h) Calculate the fitness of each particle, and update the optimal position $P_{i d}$ of each particle and the optimal position $P_{p d}$ of the population.

i) Annealing operation:

$$
\mathrm{T}=\delta \mathrm{T}
$$

j) It is judged whether the termination condition is satisfied, if it is satisfied, the search is stopped, the calculation result is output, and if it is not satisfied, the process proceeds to step e).

\subsection{Parameter setting}

\subsubsection{Setting of inertia weight}

The value of inertia weight is one of the factors influencing the result of the optimization algorithm in the process of microgrid optimization algorithm[8-9]. Its function is that the current particle inherits the size of the previous particle velocity, which will directly affect the speed of particle optimization and the position of global search. In order to improve the efficiency of the algorithm, this paper uses the method of decreasing weight. At the initial stage of the operation, that is, far away from the optimal solution position, the convergence speed is faster. In the later stage of the algorithm operation, when the optimal solution is approached, the search speed cannot be Too fast, so that the particle swarm can more accurately search for the optimal solution locally. The formula is as follows:

$$
\omega_{t}=\omega_{\max }-\left(\omega_{\max }-\omega_{\min }\right)\left(\frac{k}{K_{\max }}\right)^{2}
$$

In the formula: $\omega_{\max }$ and $\omega_{\min }$ are almost the initial value and the final value of the inertia weight respectively. After experiments, when the values of the parameters $\omega_{\max }$ and $\omega_{\min }$ are set to 0.9 and 0.4 respectively, the search ability of the particles is better. $k$ is the current number of iterations, and $K_{\max }$ is the maximum number of iterations.

\subsubsection{Settings for $c_{1}, c_{2}$}

$c_{1}$ and $c_{2}$ are learning factors, which respectively represent the learning ability of the particles in their best state and the best state of the population. In the experiment, $c_{1}$ and $c_{2}$ take 2.05 .

\subsubsection{Settings for $r_{1}, r_{2}$}

The variables $r_{1}$ and $r_{2}$ indicate that the entire search space is to be covered, so it is a random number between 0 and 1.

\subsubsection{Initial temperature setting}

The initial temperature $\mathrm{T}$ setting can directly affect the global search ability of the simulated annealing particle swarm algorithm. To improve the possibility of searching for the global optimal solution, a higher initial temperature can be set. The formula is as follows:

$$
\mathrm{T}=-\frac{f\left(P_{p d}\right)}{\ln 0.2}
$$

\subsubsection{Annealing speed setting}

In the simulated annealing particle swarm algorithm, in order to improve the probability of finding the global optimal solution, the simulated annealing process can be made as slow as possible, so the simulated annealing speed is 0.95 .

\section{Numerical simulation analysis}

In order to verify the feasibility of the above model and algorithm, this paper uses matlab simulation to analyze the actual microgrid system in the higher altitude region of remote areas, and the average is one day for the calculation cycle. Predicting the PV and wind power generation and the user load of the community on the day, PV and WT are the maximum power operation. The maximum charge and discharge power of the energy storage device is $10 \mathrm{kw}$, and the power purchased from the large power grid does not exceed $40 \%$ of the daily power load. The premise is that the large grid is fully charged, and power outage losses are not considered for the time being. The relevant parameters of SAPSO are set as: the search space dimension (that is, the unknown number) is 48 ; the number of particles is 600 ; the maximum number of iterations is 300 .

\subsection{Simulation parameter setting}

The photovoltaic power generation and wind power generation are predicted by reference to the local area light intensity and wind speed as follows: 


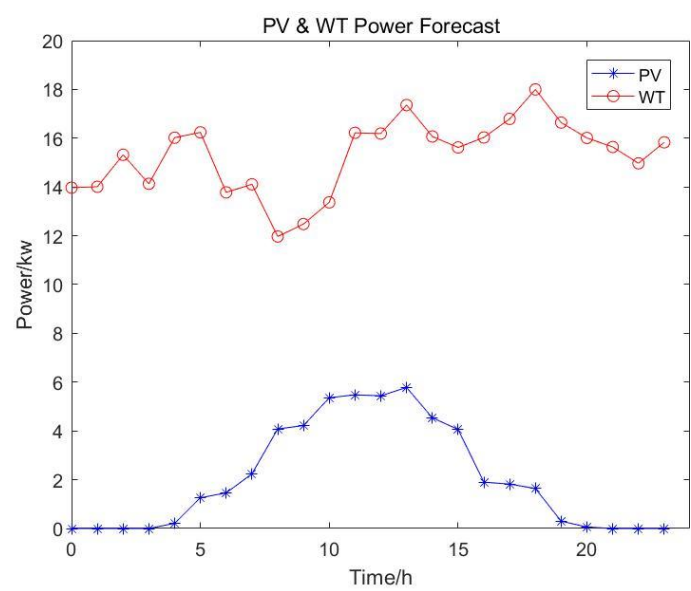

Fig. 4. PV\&WT Power Forecast

Referring to the traffic load of the community in previous years, the predicted user load is as shown in the figure:

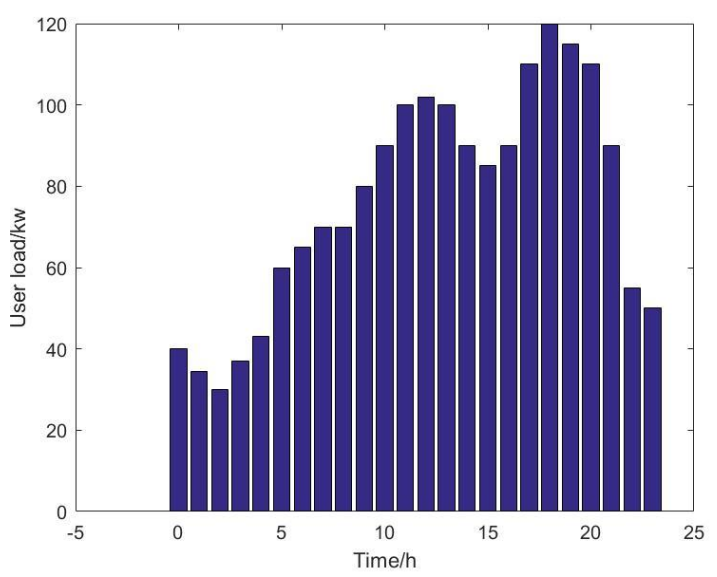

Fig. 5. Residential Electricity Load Forecast

It can be seen from the figure that the renewable energy full-state power generation still cannot meet the demand load of the residents, so the power-controlled micro-power supply needs to be powered together. The micro-power sources used in the above micro-grid model are: fuel cell (FC), micro gas turbine (MT), diesel generator (DEG) and lead acid battery (BAT). The relevant parameters are shown in Table 1. The corresponding emission factors and treatment costs of pollutant discharge types are shown in Table 2, and the local price lists for different time zones are shown in Table 3.

Table 1. Related parameters of each processing unit in the microgrid system

\begin{tabular}{|c|c|c|c|}
\hline Micro power & PV & WT & BAT \\
\hline rated power (kw) & 8 & 25 & 50 \\
\hline fuel cost (yuan/kwh) & $/$ & $/$ & $/$ \\
\hline
\end{tabular}

\begin{tabular}{|c|c|c|c|}
\hline operating costs (yuan/kwh) & 0.0096 & 0.045 & 0.045 \\
\hline output range $(\mathrm{kw})$ & $0 \sim 6$ & $0 \sim 20$ & $-10 \sim 10$ \\
\hline climbing rate $(\mathrm{kw} / \mathrm{h})$ & / & / & 20 \\
\hline Depreciation period (year) & 20 & 10 & 10 \\
\hline $\begin{array}{l}\text { Installation cost (Ten } \\
\text { thousand yuan } / \mathrm{kw})\end{array}$ & 6.65 & 2.375 & 0.087 \\
\hline Micro power & FC & MT & DEG \\
\hline rated power $(\mathrm{kw})$ & 40 & 65 & 30 \\
\hline fuel cost (yuan/kwh) & 0.199 & 0.358 & 1.152 \\
\hline operating costs (yuan/kwh) & 0.0293 & 0.128 & 0.0158 \\
\hline output range $(\mathrm{kw})$ & $3 \sim 30$ & $4 \sim 40$ & $2 \sim 15$ \\
\hline climbing rate $(\mathrm{kw} / \mathrm{h})$ & 60 & 80 & 40 \\
\hline Depreciation period (year) & 10 & 10 & / \\
\hline $\begin{array}{l}\text { Installation cost (Ten } \\
\text { thousand yuan } / \mathrm{kw})\end{array}$ & 4.275 & 1.306 & l \\
\hline
\end{tabular}

Table 2. Pollutant emission factor and treatment cost

\begin{tabular}{|c|c|c|c|}
\hline Type of pollutant & $\mathrm{NO}_{x}$ & $\mathrm{SO}_{2}$ & $\mathrm{CO}_{2}$ \\
\hline $\begin{array}{c}\text { FC emission } \\
\text { factor(kg/kw॰h) }\end{array}$ & 0.014 & 0.008 & 1.596 \\
\hline $\begin{array}{c}\text { MT emission } \\
\text { factor(kg/kw॰h) }\end{array}$ & 0.2 & 0.0036 & 1.078 \\
\hline $\begin{array}{c}\text { DEG emission } \\
\text { factor(kg/kw॰h) }\end{array}$ & 21.8 & 0.454 & 1.432 \\
\hline $\begin{array}{c}\text { Grid emission } \\
\text { factor(kg/kw॰h) }\end{array}$ & 3.6 & 4.54 & 0.997 \\
\hline $\begin{array}{c}\text { Processing } \\
\text { cost(yuan/kg) }\end{array}$ & 26.46 & 6.237 & 0.088 \\
\hline
\end{tabular}

Table 3. Local time zone electricity price list

Note:

The peak load period is: 10:00-13:00, 17:00-20:00;

The load period is 0:00-7:00, 23:00-0:00;

The rest is the load period. 


\begin{tabular}{|c|c|c|c|c|}
\hline \multirow{2}{*}{ Category } & \multirow{2}{*}{$\begin{array}{c}\text { Unified } \\
\text { electricity } \\
\text { price } \\
\text { (yuan/kwh) }\end{array}$} & \multicolumn{3}{|c|}{$\begin{array}{l}\text { Time-of-use price } \\
\text { (yuan/kwh) }\end{array}$} \\
\hline & & $\begin{array}{l}\text { Low } \\
\text { load } \\
\text { period }\end{array}$ & $\begin{array}{l}\text { Load } \\
\text { nomal } \\
\text { period }\end{array}$ & $\begin{array}{c}\text { Peak } \\
\text { load } \\
\text { period }\end{array}$ \\
\hline $\begin{array}{l}\text { Purchase } \\
\text { electricity }\end{array}$ & 0.55 & 0.28 & 0.54 & 0.83 \\
\hline $\begin{array}{c}\text { Sale of } \\
\text { electricity }\end{array}$ & 0.39 & 0.13 & 0.38 & 0.65 \\
\hline
\end{tabular}

\subsection{Running results in two algorithms in the grid-connected mode}

It can be seen from Table 2 in Table 1 above that the fuel cost and operating cost are lower than the pollutant treatment cost, so the economy and environmental protection cannot be optimized at the same time. This paper only considers the microgrid operation mode when the comprehensive benefit is optimal. Photovoltaic power generation and wind power generation equipment are fully utilized, and the remaining micro power supply working conditions and the power exchange between the microgrid and the large power grid are as shown in the following figures:
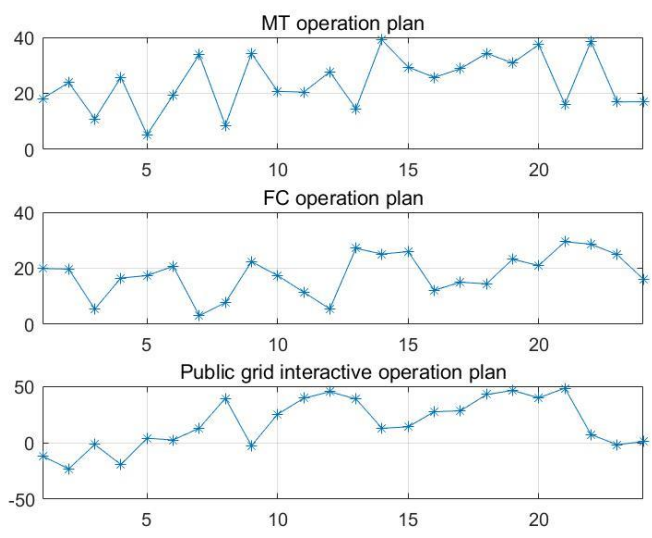

Fig. 6. Operation of micro power supplies under traditional particle swarm optimization
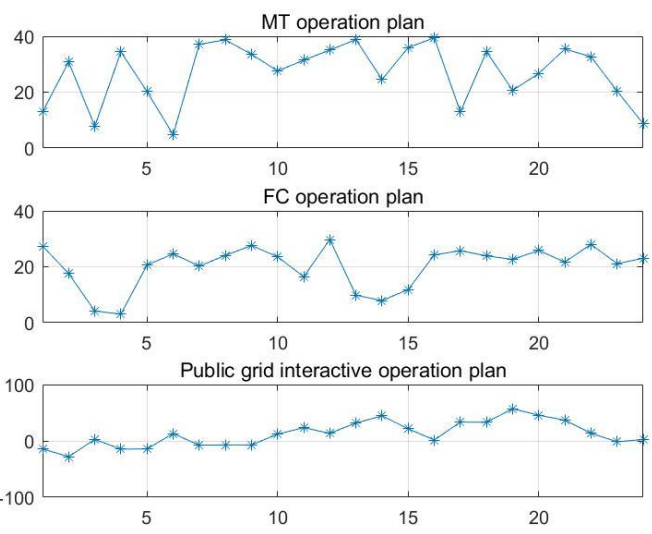

Fig. 7. Improved micro-power operation under particle swarm optimization

Under the premise of ensuring the safety and stability of the microgrid and meeting the needs of users, the 24hour results of the microgrid operation are shown in Table 4 under the model of the objective function considering comprehensive benefits:

Table 4. Comprehensive benefit cost under different algorithms

\begin{tabular}{|c|c|}
\hline algorithm & $\begin{array}{c}\text { cost } \\
\text { (yuan) }\end{array}$ \\
\hline $\begin{array}{c}\text { Traditional particle } \\
\text { swarm optimization }\end{array}$ & 40588.8 \\
\hline $\begin{array}{c}\text { Improved particle } \\
\text { swarm optimization }\end{array}$ & 37453.7 \\
\hline
\end{tabular}

As can be seen from the above chart, the particle swarm optimization algorithm based on the simulated annealing algorithm yields less cost and saves about 3,000 yuan. The cost of purchasing electricity from the public grid is lower, but it will increase the burden on the public grid. As can be seen from Figure 6 and Figure 7, the improved algorithm not only achieves lower cost, but also does not rely on the power supply of the large grid. Increased the independence of the microgrid.

\section{5 conclusion}

In this paper, the optimal scheduling problem of micro power supply operation modes in microgrid gridconnected operation mode is studied. Taking the higher altitude region in a remote area of China as an example, Using the particle swarm optimization algorithm based on simulated annealing algorithm to find the optimal scheduling scheme for the cooperation of each output unit of microgrid. Under the premise of satisfying system constraints and user needs, not only the lowest economic cost but also the best environmental benefits are considered, and a comprehensive benefit cost model with comprehensive consideration of economic environmental protection is established. The operating modes of FC and MT are tortuous, fully explaining the contradiction between micro-power sources and revealing the rationality of considering the comprehensive benefit operation mode. Finally, the simulation results show that the improved particle swarm optimization algorithm is more suitable for this model and has certain reference value.

\section{References}

1. Peng L I, Weina X U, Zeyuan Z, et al. Optimal Operation of Microgrid Based on Improved Gravitational Search Algorithm[J]. Proceedings of the CSEE, 19(2014)

2. Shuang-Xin, W. , Fang, H. , \& Heng-Jun, Z. . (2005). Economic load dispatch based on improved 
mutative scale chaotic optimization. Proceedings of the Csee, 25(24), 90-95

3. Li C , Zhang J, Li P . Multi-objective optimization model of micro-grid operation considering cost, pollution discharge and risk[J]. Zhongguo Dianji Gongcheng Xuebao/Proceedings of the Chinese Society of Electrical Engineering, 35,5(2015):10511058

4. D. Liu, N. Wang and Y. An, "Multi-targets Tracking Algorithm Based on Weighted Centroid," 2010 Second WRI Global Congress on Intelligent Systems (Wuhan, 2010, pp. 420-423)

5. L. Jianxiang et al., "The capacity optimization of hybrid energy storage system for wind power smoothing," 2014 IEEE International Conference on Mechatronics and Automation (Tianjin, 2014, pp. 2106-2110)

6. S. Cui, H. Lv, X. Wu, Y. Zhang and L. He, "Optimization of plant light source based on simulated annealing particle swarm optimization algorithm," 2018 Chinese Control And Decision Conference (CCDC) (Shenyang, 2018, pp. 700-703)

7. R. Madhumathi and R. Radhakrishnan, "A resource allocation strategy in cloud using Roulette Wheel Selection method," 2015 International Conference on Green Computing and Internet of Things (ICGCIoT) (Noida, 2015, pp. 341-345)

8. I. K. Gupta, A. Choubey and S. Choubey, "Particle swarm optimization with selective multiple inertia weights," 2017 8th International Conference on Computing, Communication and Networking Technologies (ICCCNT) (Delhi, 2017, pp. 1-6)

9. Xiao-Hui, Y., Cheng, W. , Yong-Chuan, Z. , \& Yan-Bin, Y. . (2004). A survey on application of particle swarm optimization to electric power systems. Power System Technology, 28(19), 14-19 